\title{
AAAI Conferences Calendar
}

This page includes forthcoming AAAI sponsored conferences, conferences presented by AAAI Affiliates, and conferences held in cooperation with AAAI. AI Magazine also maintains a calendar listing that includes nonaffiliated conferences at www.aaai.org/Magazine/calendar.php.

\section{AAAI Sponsored Conferences}

AAAI Spring Symposium Series. The AAAI Spring Symposium Series will be held March 25-27, 2013 at Stanford University, Stanford, California, USA

URL: www.aaai.org/Symposia/Spring/ sss13.php

Seventh International AAAI Conference on Weblogs and Social Media. ICWSM-13 will be held July 8-11, 2013 at MIT, Cambridge, MA USA

URL: www.icwsm.org/2013/

Twenty-Seventh AAAI Conference on Artificial Intelligence and TwentyFifth Innovative Applications of Artificial Intelligence Conference. AAAI13 and IAAI-13 will be held July 14-18, 2013, in Bellevue, Washington, USA

URL: www.aaai.org/aaai13

Ninth AAAI Conference on Digital Entertainment. AIIDE-13 will be held October 14-18 at Northeastern University in Boston, Massachusetts, USA. www.aaai.org/Symposia/Fall/fss13.php

AAAI Fall Symposium Series. The AAAI Fall Symposium Series will be held November 15-17 at the Westin Arlington Gateway in Arlington, Virginia, near Washington, DC USA.

www.aaai.org/Symposia/Fall/fss13.php

Twenty-Eighth AAAI Conference on Artificial Intelligence. AAAI-14 will be held July 27-31, 2014, in Quebec City, Quebec, Canada

URL: www.aaai.org/aaai14
Twenty-Sixth Innovative Applications of Artificial Intelligence Conference. IAAI-14 will be held July 27-31, 2014, in Quebec City, Quebec, Canada

URL: www.aaai.org/iaai14

\section{Conferences Held by AAAI Affiliates}

Twenty-Sixth International FLAIRS Conference. FLAIRS 2013 will be held May 22-24, 2013 at the TradeWinds Island Resort, St. Pete Beach, FL

$U R L$ : www.flairs-26.info

Twenty-Third International Conference on Automated Planning and Scheduling. ICAPS 2013 will be held 10-14 June, 2013 in Rome, Italy

Twenty-Third International JointConference on Artificial Intelligence. IJCAI-13 will be held August 39, 2013 in Beijing, China

URL: ijcai.org

\section{Conferences Held in Cooperation with AAAI}

Fourteenth International Conference on Artificial Intelligence and Law. ICAIL 2013 will be held June 1014, 2013, in Rome, Italy

URL:icail2013.ittig.cnr.it

Fourth International Conference on Computational Creativity. ICCC 2013 will be held June 12-14, 2013, in Sydney, Australia

URL:

computationalcreativity.net/iccc2013
Twenty-Sixth International Conference on Industrial Engineering and Other Applications of Artificial Intelligence and Expert Systems. IEA/AIE-13 will be held June 17-21, 2013, in Amsterdam, The Netherlands

URL:iea-aie2013.few.vu.nl

Fifteenth International Conference on Enterprise Information Systems. ICEIS 2013 will be held July 3-7, 2013 in Angers, France

URL: www.iceis.org

Twenty-First Conference on CaseBased Reasoning. ICCBR 2013 will be held July 8-11, 2013 in Saratoga Springs, New York USA

$U R L:$ www.iccbr.org

Sixth Annual Symposium on Combinatorial Search. SoCS 2013 will be held July 11-13, 2013, Seattle, Washington, USA

$U R L$ : socs13.search-conference.org

Tenth International Conference on Informatics in Control, Automation and Robotics. ICINCO 2013 will be held July 28-31, 2013 in Reykjavík, Iceland

URL: www.icinco.org

Thirty-Fifth Annual Conference of the Cognitive Science Society. COGSCI 2013 will be held July 31 August 3, 2013 in Berlin, Germany

URL: cognitivesciencesociety.org/conference 2013

The Sixth Conference on Artificial General Intelligence. AGI-13 will be held July 31 - August 3, 2013, in Beijing, China

URL: agi-conf.org/2013

The Thirteenth International Conference on Intelligent Virtual Agents. IVA 2013 will be held August 29-31, 2013, in Edinburgh, UK

URL: www.cstr.ed.ac.uk/iva2013

Fifth Biannual Humaine Association Conference on Affective Computing and Intelligent Interaction. ACII 2013 will be held September 2 - 5, 2013, in Geneva, Switzerland.

URL: www.acii2013.org 